\title{
ON THE STRICT ARNOLD CHORD PROPERTY AND COISOTROPIC SUBMANIFOLDS OF COMPLEX PROJECTIVE SPACE
}

\author{
FABIAN ZiLTENER \\ Utrecht University, Mathematics Department, \\ Budapestlaan 6, 3584CD Utrecht, The Netherlands \\ E-MAIL:F.ZILTENER@UU.NL
}

Let $\alpha$ be a contact form on a manifold $M$, and $L \subseteq M$ a closed Legendrian submanifold. I prove that $L$ intersects some characteristic for $\alpha$ at least twice if all characteristics are closed and of the same period, and $\alpha$ embeds nicely into the product of $\mathbb{R}^{2 n}$ and an exact symplectic manifold. As an application of the method of proof, the minimal action of a regular closed coisotropic submanifold of complex projective space is at most $\pi / 2$. This yields an obstruction to presymplectic embeddings, and in particular to Lagrangian embeddings.

Contents

1. Main results

1.1. The strict chord property

1.2. Minimal action of a regular coisotropic submanifold of complex projective space

1.3. Related work

1.4. Acknowledgments

2. Proof of Theorem 1 (strict chord property)

3. Proof of Proposition 3 (contact form)

4. Proof of Theorem [6 (minimal action) and of Corollary 8 (presymplectic embedding)

Appendix A. Lifting paths

Appendix B. Coisotropic subspaces of presymplectic vector spaces

References 


\section{Main results}

1.1. The strict chord property. Let $M$ be a manifold (possibly noncompact or with boundary) and $\alpha$ a contact form on $M$. We say that $(M, \alpha)$ has the strict chord property iff for every nonempty closed 1 Legendrian submanifold $L \subseteq M$ there exists a characteristic for $\alpha$ 2 that intersects $L$ at least twice. To explain this terminology, note that parametrizing part of such a characteristic, we obtain a strict Reeb chord, i.e., an integral curve of the Reeb vector field that starts and ends at different points of $L$.

Such chords arise in classical mechanics as libration motions, i.e., oscillations of a mechanical system between two rest points, see Ci. p. 118]. The present article is concerned with the following problem.

Problem (strict chord problem). Find conditions on $(M, \alpha)$ under which it has the strict chord property.

In [Ar, p. 11] V. I. Arnol'd conjectured that for $n \geq 2$ any contact form on $S^{2 n-1}$ inducing the standard structure has the strict chord property. The main result of this article roughly is that this property holds for every contact form on a manifold if all its characteristics are closed and of the same period and the contact form nicely embeds into the product of $\mathbb{R}^{2 n}$ and an exact symplectic manifold. In particular, this confirms Arnol'd's conjecture for the standard form on $S^{2 n-1}$.

To state the result, let $M$ be a manifold and $\alpha$ a contact form on $M$. The period of a closed characteristic $C$ for $\alpha$ is the number

$$
\left|\int_{C} \iota^{*} \alpha\right|
$$

where $\iota: C \rightarrow M$ denotes the inclusion and we equip $C$ with either orientation. We denote by $q^{1}, p_{1}, \ldots, q^{n}, p_{n}$ the standard coordinates of $\mathbb{R}^{2 n}$, and define

$$
\lambda_{0}:=\frac{1}{2} \sum_{i=1}^{n}\left(q^{i} d p_{i}-p_{i} d q^{i}\right) .
$$

Theorem 1 (strict chord property). The pair $(M, \alpha)$ has the strict chord property if all characteristics for $\alpha$ are closed and of equal period $T$, and there exist a manifold $W$ together with a one-form $\lambda$, an integer

\footnotetext{
${ }^{1}$ This means "compact and without boundary".

${ }^{2}$ This means a leaf of the foliation determined by the integrable distribution $\operatorname{ker}\left(d \alpha: T M \rightarrow T^{*} M\right)$ on $M$, i.e., an unparametrized Reeb trajectory.
} 
$n \geq \frac{1}{2} \operatorname{dim} W+2$, and an embedding $\varphi: M \rightarrow \mathbb{R}^{2 n} \times W$, such that $d \lambda$ is a geometrically bounded ${ }^{3}$ symplectic form, and

$$
\begin{gathered}
\operatorname{dim} M=2 n+\operatorname{dim} W-1, \\
\varphi(M) \subseteq \bar{B}^{2 n}(T) \times W, \\
\varphi^{*}\left(\lambda_{0} \oplus \lambda\right)=\alpha .
\end{gathered}
$$

Here $\bar{B}^{2 n}(a)$ denotes the closed ball in $\mathbb{R}^{2 n}$ of radius $\sqrt{a / \pi}$.

The proof of Theorem 1 is based on a result by Yu. Chekanov, which implies that the displacement energy of a closed Lagrangian submanifold in a geometrically bounded symplectic manifold is at least the minimal symplectic action of the Lagrangian.

Assuming by contradiction that there is no strict Reeb chord, such a Lagrangian is constructed from the given Legendrian submanifold by moving it with the Reeb flow. This technique is a variation on the approach used by K. Mohnke in [Mo. (In that article the Lagrangian was obtained by moving the Legendrian both with the Reeb flow and with the Liouville flow.)

A crucial ingredient of the proof is the fact that the displacement energy of a compact subset $X$ of the closed unit ball $\bar{B}^{2 n}$ is strictly less than $\pi$, provided that $X$ does not contain the unit sphere. (See Lemma 12 below.)

Theorem 1 has the following immediate application. We denote by $\iota: S^{2 n-1} \rightarrow \mathbb{R}^{2 n}$ the inclusion.

Corollary 2 (sphere). For $n \geq 2$ the standard contact form $\alpha_{0}:=\iota^{*} \lambda_{0}$ on $S^{2 n-1}$ has the strict chord property.

More examples are obtained by the following construction. By an exact Hamiltonian $S^{1}$-manifold we mean a triple consisting of a smooth manifold $W$, a smooth $S^{1}$-action $\rho$ on $W$, and an $\rho$-invariant one-form $\lambda$ on $W$, such that $d \lambda$ is non-degenerate. We fix such a triple and numbers $c \in(0, \infty)$ and $n \in \mathbb{N} \cup\{0\}$. We denote by $X$ the vector field

\footnotetext{
${ }^{3}$ We call a symplectic form $\omega$ on a manifold $W$ geometrically bounded iff there exists an $\omega$-compatible almost complex structure $J$ on $W$ such that the metric $\omega(\cdot, J \cdot)$ is complete with bounded sectional curvature and injectivity radius bounded away from 0 . Examples are closed symplectic manifolds, cotangent bundles of closed manifolds, and symplectic vector spaces.
} 
generated by $\rho .{ }^{4}$ We define

(6) $M:=\left\{\left(x_{0}, x\right) \in\left(\bar{B}^{2 n}(2 \pi c) \backslash\{0\}\right) \times W \mid H_{0}\left(x_{0}\right)+H(x)=c\right\}$.

We denote by

$$
\iota: M \rightarrow \widetilde{W}:=\mathbb{R}^{2 n} \times W
$$

the inclusion.

Proposition 3 (contact form). The set $M$ is a (smoothly embedded) hypersurface in $\widetilde{W}^{5}$, and

$$
\alpha:=\iota^{*}\left(\lambda_{0} \oplus \lambda\right)
$$

is a contact form on $M$ all of whose characteristics are closed and of period $2 \pi c$.

Corollary 4 (strict chord property). If $(W, d \lambda)$ is geometrically bounded and $n \geq \frac{1}{2} \operatorname{dim} W+2$ then $(M, \alpha)$ has the strict chord property.

Proof of Corollary 4. This follows from Theorem 1, using Proposition 3 and the facts that $\left(\mathbb{R}^{2 n}, \omega_{0}\right)$ is geometrically bounded, geometric boundedness is invariant under products, and that conditions (1, 2, 3) are satisfied with $\varphi:=\iota$.

Example 5. Let $X$ be a manifold. We define $W:=T^{*} X$ and $\lambda$ to be the canonical one-form on $W$. We fix a smooth $S^{1}$-action $\sigma$ on $X$ and define

$$
\rho: S^{1} \rightarrow \operatorname{Diff}(W), \quad \rho(z)(q, p):=\left(\sigma_{z}(q), p d \sigma_{z}(q)^{-1}\right),
$$

where $\sigma_{z}:=\sigma(z)$. The triple $(W, \rho, \lambda)$ is an exact Hamiltonian $S^{1}$ manifold, and $(W, d \lambda)$ is geometrically bounded. Hence by Corollary 4 the pair $(M, \alpha)$, defined as in (6), 7), is a contact manifold that has the strict chord property, provided that $n \geq \frac{1}{2} \operatorname{dim} W+2$.

Remark. There exist contact forms on closed manifolds that do not have the strict chord property. The simplest example is the standard contact form on $S^{1}$.

\footnotetext{
${ }^{4}$ This is the infinitesimal action of the element $1 \in \mathbb{R}=\operatorname{Lie} S^{1}$, where we identify $S^{1}$ with $\mathbb{R} / 2 \pi \mathbb{Z}$.

${ }^{5} M$ may have a boundary.
} 
Another example, which is taken from [Mo, goes as follows. We denote by $\gamma$ the standard angular form on $S^{1}$ that integrates to $2 \pi$. Consider the contact one-form on $M:=S^{1} \times S^{2}$ given by

$$
\alpha:=x_{1} \gamma+\frac{1}{2}\left(x_{2} d x_{3}-x_{3} d x_{2}\right),
$$

where $x \in S^{2} \subseteq \mathbb{R}^{3}$. Each Legendrian loop $S^{1} \times\left\{\left(0, x_{2}, x_{3}\right)\right\}$ intersects each Reeb orbit $\{z\} \times\left\{x_{1}=0\right\}$ (with $z \in S^{1}$ ) only once.

1.2. Minimal action of a regular coisotropic submanifold of complex projective space. The bound on the displacement energy of a compact subset of $\bar{B}^{2 n}$, which is used in the proof of Theorem 1. and a coisotropic version of Chekanov's theorem have the following application. Let $(M, \omega)$ be a symplectic manifold and $N \subseteq M$ a coisotropic submanifold. ${ }^{6}$

We denote by $N_{\omega}$ the set of all isotropic (or characteristic) leaves of $N^{7}$, and by $\mathbb{D} \subseteq \mathbb{R}^{2}$ the closed unit disk. We define the action spectrum and the minimal action (or area) of $N$ to be

$$
\begin{gathered}
S(N):=S(M, N):=S(M, \omega, N):= \\
\left\{\int_{\mathbb{D}} u^{*} \omega \mid u \in C^{\infty}(\mathbb{D}, M): \exists F \in N_{\omega}: u\left(S^{1}\right) \subseteq F\right\}, \\
A(N):=A(M, N):=A(M, \omega, N):= \\
\inf (S(N) \cap(0, \infty)) \in[0, \infty] .
\end{gathered}
$$

Remarks. - In the case $N=M$ we have

$$
A(M)=\inf \left(\left\{\int_{S^{2}} u^{*} \omega \mid u \in C^{\infty}\left(S^{2}, M\right)\right\} \cap(0, \infty)\right) .
$$

(See [SZ, Lemma 29].)

- If $N$ is Lagrangian then

$$
A(N)=\inf \left(\left\{\int_{\mathbb{D}} u^{*} \omega \mid u \in C^{\infty}(\mathbb{D}, M): u\left(S^{1}\right) \subseteq N\right\} \cap(0, \infty)\right) .
$$

${ }^{6}$ This means that for every $x \in N$ the symplectic complement

$$
T_{x} N^{\omega}:=\left\{v \in T_{x} M \mid \omega(v, w)=0, \forall w \in T_{x} N\right\}
$$

is contained in $T_{x} N$.

${ }^{7}$ The set

$$
T N^{\omega}=\left\{(x, v) \mid x \in N, v \in T_{x} N^{\omega}\right\} \subseteq T N
$$

is an involutive distribution on $N$. Hence by Frobenius' theorem it gives rise to a foliation on $N$. Its leaves are called the isotropic leaves of $N$. 
We call $N$ regular iff there exists a manifold structure ${ }^{8}$ on $N_{\omega}$, such that the canonical projection $\pi: N \rightarrow N_{\omega}$ is a smooth submersion. ${ }^{9}$ Examples are Lagrangian submanifolds, $N=M$, and the sphere $N=$ $S^{2 n-1} \subseteq M=\mathbb{R}^{2 n}$. (For further examples see [Zi $]$.) Regularity is invariant under taking products.

Let $n \in \mathbb{N}=\{1,2, \ldots\}$. We equip the complex projective space $\mathbb{C P}^{n}$ with the Fubini-Study form $\omega_{\mathrm{FS}}{ }^{10}$

Theorem 6 (minimal action). Let $(M, \omega)$ be a geometrically bounded symplectic manifold, and $\emptyset \neq N$ a regular closed coisotropic submanifold of $\mathbb{C P}^{n} \times M$ of dimension less than $2 n$. Then

$$
A(N) \leq \frac{\pi}{2}
$$

Remarks. - The hypothesis that $\operatorname{dim} N<2 n$ cannot be dropped. Otherwise,

$$
\begin{gathered}
M:=\{\mathrm{pt}\}, \quad N:=\mathbb{C P}^{n}, \quad \text { and } \\
M:=\mathbb{C P}^{n}, \quad \omega:=-\omega_{\mathrm{FS}}, \quad N:=\left\{(x, x) \mid x \in \mathbb{C P}^{n}\right\}
\end{gathered}
$$

are counterexamples.

- The hypothesis that $N$ be regular cannot be dropped. To see this, let $n \geq 2$. Then there exists a closed hypersurface $N_{0} \subseteq \mathbb{R}^{2 n}$ without any closed characteristic, see $\mathbf{G G}$ and references therein. ${ }^{11}$ By shrinking $N_{0}$ with a homothety and using a Darboux chart, we otain a hypersurface $N$ inside $\mathbb{C P}^{n}$ with the same property. It satisfies $A(N)=\pi$ (but is not regular).

Corollary 7 (minimal action). Let $(M, \omega)$ be a geometrically bounded symplectic manifold, such that $\operatorname{dim} M<2 n$. Then the minimal action of a closed nonempty Lagrangian submanifold of $\mathbb{C P}^{n} \times M$ is bounded above by $\pi / 2$.

Proof. This follows from Theorem 6 and the fact that every Lagrangian submanifold is regular.

\footnotetext{
${ }^{8}$ The induced topology is by definition Hausdorff and second countable.

${ }^{9}$ In this case the symplectic quotient of $N$ is well-defined in the sense that this manifold structure is unique and there exists a unique symplectic form on $N_{\omega}$ that pulls back to $\iota^{*} \omega$ under $\pi$. Here $\iota: N \rightarrow M$ denotes the inclusion. The manifold structure on $N_{\omega}$ induces the quotient topology on this set.

${ }^{10}$ This form is normalized in such a way that the area of a projective line is $\pi$.

${ }^{11}$ In the case $n \geq 3$ this hypersurface may be chosen to be smooth, but for $n=2$ the hypersurface constructed in $\mathbf{G G}$ is only $C^{2}$.
} 
Remark. By Corollary 7 there is no exact Lagrangian submanifold of $\mathbb{C P}^{n} \times M$. By this we mean a Lagrangian submanifold with minimal action equal to $\pi$.

To explain a further application of Theorem 6, recall that a presymplectic form on a manifold $M$ is a closed two-form $\omega$ on $M$, such that

$$
\operatorname{corank} \omega_{x}:=\operatorname{dim}\left(T_{x} M\right)^{\omega}
$$

does not depend on $x \in M .{ }^{12}$ Let $\omega$ be such a form. The set

$$
T M^{\omega}=\left\{(x, v) \mid x \in N, v \in T_{x} M^{\omega}\right\} \subseteq T M
$$

is an involutive distribution on $M$. Hence by Frobenius' theorem it gives rise to a foliation on $M$. We denote by $M_{\omega}$ the set of its leaves. We call $(M, \omega)$ regular iff there exists a manifold structure on $M_{\omega}$ for which the canonical projection $\pi: M \rightarrow M_{\omega}$ is a smooth submersion.

A presymplectic embedding of a presymplectic manifold into another one is by definition a smooth embedding that intertwines the two presymplectic forms.

Corollary 8 (presymplectic embedding). Let $n \in \mathbb{N}$ and $(M, \omega)$ be a geometrically bounded symplectic manifold, such that

$$
\int_{S^{2}} u^{*} \omega \in \pi \mathbb{Z}, \quad \forall u \in C^{\infty}\left(S^{2}, M\right) .
$$

Let $\left(M^{\prime}, \omega^{\prime}\right)$ be a nonempty closed regular presymplectic manifold, such that every isotropic leaf of $M^{\prime}$ is simply connected, and

$$
\begin{gathered}
\operatorname{dim} M^{\prime}+\operatorname{corank} \omega^{\prime}=2 n+\operatorname{dim} M, \\
\operatorname{dim} M^{\prime}<2 n .
\end{gathered}
$$

Then $\left(M^{\prime}, \omega^{\prime}\right)$ does not presymplectically embed into the symplectic manifold $\left(\mathbb{C P}^{n} \times M, \omega_{\mathrm{FS}} \oplus \omega\right)$.

Example. Let $F$ be a simply-connected closed manifold of positive dimension and $(X, \sigma)$ a closed symplectic manifold. By Corollary 8 the presymplectic manifold $(X \times F, \sigma \oplus 0)$ does not embed into $\left(\mathbb{C P}^{n}, \omega_{\mathrm{FS}}\right)$, where $n:=\frac{1}{2} \operatorname{dim} X+\operatorname{dim} F$.

Corollary 8 will be proved in Section 4 . It has the following immediate application.

\footnotetext{
${ }^{12}$ Here

$$
\left(T_{x} M\right)^{\omega}=\left\{v \in T_{x} M \mid \omega(v, w)=0, \forall w \in T_{x} M\right\} .
$$
}


Corollary 9 (Lagrangian embedding). Let $n \in \mathbb{N}$ and $(M, \omega)$ be a geometrically bounded symplectic manifold such that (10) holds and $\operatorname{dim} M<2 n$. Then no simply-connected closed manifold embeds into $\mathbb{C P}^{n} \times M$ in a Lagrangian way.

1.3. Related work. V. I. Arnol'd observed in $\mathbf{A r}$ that the strict chord property for $\left(S^{3}, \alpha_{0}\right)$ follows from an elementary argument. In Giv, Corollary 1] A. B. Givental' proved that there exists a Reeb chord between every pair of Legendrian submanifolds of $\mathbb{R} \mathrm{P}^{2 n-1}$ with the standard contact form, if they are isotopic via Legendrian submanifolds to the standard $\mathbb{R} \mathrm{P}^{n-1}$.

In Ch1 Yu. Chekanov provided lower bounds on the number of critical points of a quasi-function, i.e., a Legendrian submanifold of the 1-jet bundle of a manifold that is smoothly homotopic (via Legendrians) to the zero section. These points correspond to Reeb chords between the zero section and the Legendrian.

C. Abbas Ab1, Ab2, Ab3 proved the strict chord property for certain Legendrian knots in tight closed contact 3-manifolds.

We say that a contact form $\alpha$ on a manifold $M$ has the chord property iff every closed Legendrian intersects some characteristic for $\alpha$ at least twice or it intersects some closed characteristic (i.e., periodic Reeb orbit). Note that this property is trivially satisfied iff all characteristics are closed.

Consider now a contact manifold $(M, \xi)$ that arises as the boundary of a compact Stein manifold, and $\alpha$ a contact form on $M$ inducing $\xi$. In [Mo, Theorem 2] K. Mohnke proved that $\alpha$ has the chord property. It follows that a nonempty closed Legendrian submanifold of $M$ admits a strict Reeb chord if it does not intersect any closed characteristic for $\alpha$. Intuitively such Legendrian submanifolds are generic, provided that $\operatorname{dim} M \geq 3$ and that $\alpha$ has only countably many closed Reeb orbits.

In [Ci] K. Cieliebak proved that Legendrian spheres in the boundaries of certain subcritical Weinstein domains intersect some characteristic for $\alpha$ at least twice.

Let $U \subseteq \mathbb{R}^{2 n}$ be a bounded star-shaped domain with smooth boundary and $\emptyset \neq L \subseteq \partial U$ a closed Legendrian submanifold of nonpositive curvature. The last condition means that $L$ that admits a Riemannian metric of nonpositive sectional curvature. In the recent preprint [CM] K. Cieliebak and K. Mohnke proved that $L$ possesses a Reeb chord of length bounded above by the (toroidal) Lagrangian capacity of $U$, see [CM, Corollary 1.12]. Using [CM, Corollary 1.3], they deduced that 
$L$ admits a Reeb chord of length bounded above by $\pi / n$, if $n \geq 2$, and $U=B_{1}^{2 n}$, i.e., $\partial U$ is the unit sphere.

As explained in [CM] after Corollary 1.13, it follows that there exists no exact Lagrangian embedding into $\mathbb{C P}^{n}$ of a closed manifold $\emptyset \neq X$ of nonpositive curvature. (Corollary 7 is a stabilized version of this without the nonpositive curvature assumption.)

K. Cieliebak and K. Mohnke also proved that for $S \subseteq \mathbb{R}^{2 n}$ sufficiently $C^{1}$-close to the unit sphere, every closed Legendrian submanifold $\emptyset \neq$ $L \subseteq S$ of nonpositive curvature possesses a strict Reeb chord, see [CM, Corollary 1.15].

A powerful tool for finding Reeb chords is Legendrian contact homology. Based on work by Y. Eliashberg, A. Givental, H. Hofer [EGH] and $\mathrm{Yu}$. Chekanov Ch3, this homology was developed by F. Bourgeois, T. Ekholm, J. B. Etnyre, J. Sabloff, M. Sullivan, and others, see [EESu, EESa, BEE, Ek] and references therein.

Using embedded contact homology, M. Hutchings and C. Taubes HT1, HT2 proved that every contact form on a closed 3-manifold has the chord property. Further results are contained in [Sa1, Sa2, Rit, Me.

In [Se, Theorem 3.1] P. Seidel proved that if a closed manifold $X$ embeds into $\mathbb{C P}^{n}$ in a Lagrangian way then $H^{1}(X, \mathbb{Z} /(2 n+2)) \neq 0$. In particular, $X$ is not simply-connected. Corollary 8 extends the latter statement to presymplectic embeddings into $\mathbb{C P}^{n} \times M$.

P. Biran and K. Cieliebak [BC1, BC2] generalized Seidel's result in

various ways. In the case $\int_{S^{2}} u^{*} \omega=0$, for every $u \in C^{\infty}\left(S^{2}, M\right)$, Corollary 9 follows from their results. Further references about results on the topology of Lagrangian embeddings are provided in [BC1, BC2].

1.4. Acknowledgments. I would like to thank the anonymous referee for his/ her useful comments. I would also like to thank K. Cieliebak and $\mathrm{K}$. Mohnke for making me aware of the application regarding the minimal action of a Lagrangian submanifold of $\mathbb{C P}^{n}$ (Corollary 7).

\section{Proof of Theorem 1 (strict chord property)}

The proof of Theorem 1 is based on the following construction. Let $M, \alpha, T, W, \lambda, n, \varphi$ be as in the hypothesis of Theorem 1 , and $L \subseteq M$ a nonempty closed Legendrian submanifold. We construct a Lagrangian immersion in $\mathbb{R}^{2 n} \times W$ by flowing $L$ with the Reeb flow. It will follow from Theorem 11 and Lemma 12 below that this immersion is not injective. This means that $L$ admits a strict Reeb chord. 
We identify

$$
S^{1} \cong \mathbb{R} / T \mathbb{Z}
$$

and denote by $R$ the Reeb vector field on $M$ w.r.t. $\alpha$, and by

$$
\psi: S^{1} \times M \rightarrow M
$$

its flow. This map is welldefined, since by hypothesis all Reeb orbits of $\alpha$ are closed and of period $T$. We write

$$
\widetilde{W}:=\mathbb{R}^{2 n} \times W, \quad \widetilde{\lambda}:=\lambda_{0} \oplus \lambda, \quad \widetilde{\omega}:=d \widetilde{\lambda},
$$

and denote by $\iota: S^{1} \times L \rightarrow S^{1} \times M$ the inclusion. We define

$$
f:=\varphi \circ \psi \circ \iota: S^{1} \times L \rightarrow \widetilde{W} \text {. }
$$

Lemma 10. The map $f$ is a Lagrangian immersion w.r.t. $\widetilde{\omega}$.

Proof of Lemma 10. Since $\psi$ is the flow of $R$, we have

$$
d \psi(z, x)\left(T_{z} S^{1} \times\{0\}\right)=\mathbb{R} R_{\psi(z, x)}, \quad \forall(z, x) \in S^{1} \times M .
$$

We show that $f$ is an immersion. Since $L$ is Legendrian, we have $T L \subseteq \operatorname{ker} \alpha$. Since the Reeb flow $\psi_{z}:=\psi(z, \cdot)$ preserves $\alpha$, it preserves ker $\alpha$, for every $z \in S^{1}$. It follows that $d \psi(\{0\} \times T L) \subseteq \operatorname{ker} \alpha$. Let $(z, x) \in S^{1} \times L$. Using (15) and the fact $\alpha(R) \equiv 1$, it follows that

$$
d \psi(z, x)\left(\{0\} \times T_{x} L\right) \cap d \psi(z, x)\left(T_{z} S^{1} \times\{0\}\right)=\{0\} .
$$

Since $\psi$ is a flow, $d \psi_{z}(x)$ is injective. It follows from (15) and the fact $R \neq 0$ that $d(\psi(\cdot, x))(z)$ is injective. Combining this with (16), it follows that

$$
d \psi(z, x): T_{(z, x)}\left(S^{1} \times L\right) \rightarrow T_{\psi(z, x)} M
$$

is injective. Using (14) and that $\varphi$ is an immersion, it follows that the same holds for $f$, as claimed.

We show that $f$ is isotropic. We define $\omega:=d \alpha$. The equalities (3), 14) and $\widetilde{\omega}=d \widetilde{\lambda}$ imply that

$$
f^{*} \widetilde{\omega}=d \iota^{*} \psi^{*} \varphi^{*} \widetilde{\lambda}=d \iota^{*} \psi^{*} \alpha=\iota^{*} \psi^{*} \omega .
$$

Therefore it suffices to show that $\psi^{*} \omega$ vanishes on pairs of vectors in $T\left(S^{1} \times L\right)$ (over the same point). To see this, note that for every $z \in S^{1}$ the Reeb flow $\psi_{z}: M \rightarrow M$ preserves $\omega$, since it preserves $\alpha$. Since $L$ is Legendrian, it is isotropic w.r.t. $\omega$. It follows that $\psi^{*} \omega$ vanishes on pairs of vectors in $\{0\} \times T L$.

The equalities (15) and $\mathbb{R} R=$ ker $\omega$ imply that $\psi^{*} \omega$ vanishes on each pair of vectors in $T\left(S^{1} \times L\right)$, of which at least one lies in $T S^{1} \times\{0\}$. 
It follows that $\psi^{*} \omega$ vanishes on all pairs of vectors in $T\left(S^{1} \times L\right)$. This shows that $f$ is isotropic.

Equality (11) implies that the domain of $f$ has dimension equal to $\frac{1}{2} \operatorname{dim} \widetilde{W}$. It follows that $f$ is a Lagrangian immersion, as claimed. This proves Lemma 10.

The proof that the map $f$ is not injective, is based on the next result, which is due to $\mathrm{Yu}$. Chekanov. Let $(M, \omega)$ be a symplectic manifold. We denote by $\mathcal{H}(M, \omega)$ the set of all functions $H \in C^{\infty}([0,1] \times M, \mathbb{R})$ whose Hamiltonian time $t$ flow $\varphi_{H}^{t}: M \rightarrow M$ exists and is surjective, for every $t \in[0,1] .{ }^{13}$ We define the Hofer norm

$$
\|\cdot\|: \mathcal{H}(M, \omega) \rightarrow[0, \infty], \quad\|H\|:=\int_{0}^{1}\left(\sup _{M} H^{t}-\inf _{M} H^{t}\right) d t,
$$

and the displacement energy of a subset $X \subseteq M$ to be

$$
\begin{gathered}
e(X):=e(M, X):=e(M, \omega, X):= \\
\inf \left\{\|H\| \mid H \in \mathcal{H}(M, \omega): \varphi_{H}^{1}(X) \cap X=\emptyset\right\} 14
\end{gathered}
$$

Let $L \subseteq M$ be a Lagrangian submanifold. We denote by $\mathbb{D} \subseteq \mathbb{R}^{2}$ the closed unit disk. The minimal symplectic action (or area) of $L$ is defined to be

$$
\begin{gathered}
A(M, \omega, L):= \\
\inf \left(\left\{\int_{\mathbb{D}} u^{*} \omega \mid u \in C^{\infty}(\mathbb{D}, M): u\left(S^{1}\right) \subseteq L\right\} \cap(0, \infty)\right) \in[0, \infty] .
\end{gathered}
$$

Theorem 11 (displacement energy of a Lagrangian). If $(M, \omega)$ is geometrically bounded and $L$ is closed then

$$
e(L) \geq A(L) .
$$

Proof. This follows from the Main Theorem in [Ch2].

Another key ingredient in the proof that $f$ (defined as in (14)) is not injective, is the following lemma. Let $n \in \mathbb{N}$. We denote by $\omega_{0}$ the standard symplectic form on $\mathbb{R}^{2 n}$.

\footnotetext{
${ }^{13}$ The time $t$ flow of a time-dependent vector field on a manifold $M$ is always an injective smooth immersion on its domain of definition. Hence if it is everywhere well-defined and surjective then it is a diffeomorphism of $M$.

${ }^{14}$ Alternatively, one can define a displacement energy, using only functions $H$ with compact support. However, it seems more natural to allow for all functions in $\mathcal{H}(M, \omega)$.
} 
Lemma 12 (bound on displacement energy). Let $a>0$ and $X$ be a compact subset of the closed ball $\bar{B}^{2 n}(a)$, which does not contain $S^{2 n-1}(a)$, the sphere in $\mathbb{R}^{2 n}$ of radius $\sqrt{a / \pi}$. Then

$$
e\left(\mathbb{R}^{2 n}, X\right)<a .
$$

Proof of Lemma 12. W.l.o.g. we may assume that $a=\pi$. Since $X$ does not contain $S^{2 n-1}$, there exists an orthogonal linear symplectic $\operatorname{map} \Psi: \mathbb{R}^{2 n} \rightarrow \mathbb{R}^{2 n}$, such that $(1,0, \ldots, 0) \notin \Psi(X)$. We denote

$$
Y_{c}:=\{(q, p) \in \mathbb{D} \mid q \leq c\} .
$$

Since $\Psi(X)$ is compact and contained in $\bar{B}^{2 n}$, there exists $c<1$, such that

$$
\Psi(X) \subseteq Y_{c} \times \mathbb{R}^{2 n-2}
$$

We have

$$
\begin{aligned}
e\left(\mathbb{R}^{2 n}, X\right) & =e\left(\mathbb{R}^{2 n}, \Psi(X)\right) \\
& \leq e\left(\mathbb{R}^{2 n}, Y_{c} \times \mathbb{R}^{2 n-2}\right) \\
& \leq e\left(\mathbb{R}^{2}, Y_{c}\right) \\
& =\operatorname{area}\left(Y_{c}\right) \\
& <\pi \\
& =a .
\end{aligned}
$$

The fourth step follows from a concrete construction of a Hamiltonian diffeomorphism that displaces $Y_{c}$ or from a Moser type argument. This proves (17) and hence Lemma 12.

Proof of Theorem 11. Let $M, \alpha, T, W, \lambda, n, \varphi$ be as in the hypothesis of this theorem, and $L \subseteq M$ a nonempty closed Legendrian submanifold. By hypothesis all characteristics for $\alpha$ are closed, i.e., all Reeb orbits are periodic. Furthermore, their periods are all equal to $T$. We identify $S^{1} \cong \mathbb{R} / T \mathbb{Z}$ and define $\psi, \widetilde{W}, \widetilde{\lambda}, \widetilde{\omega}, f$ as in (12]13]14).

Claim 1. The map $f$ is not injective.

Proof of Claim 1. We denote

$$
\widetilde{L}:=f\left(S^{1} \times L\right),
$$

and by

$$
\mathrm{pr}_{1}: \widetilde{W} \rightarrow \mathbb{R}^{2 n}
$$


the projection onto the first factor. The hypotheses (1) and $n \geq$ $\frac{1}{2} \operatorname{dim} W+2$ imply that

$$
\operatorname{dim}\left(S^{1} \times L\right)=1+\frac{\operatorname{dim} M-1}{2}=n+\frac{1}{2} \operatorname{dim} W \leq 2 n-2 .
$$

Hence by Sard's theorem, it follows that

$$
S^{2 n-1}(T) \nsubseteq \operatorname{pr}_{1} \circ f\left(S^{1} \times L\right)=\operatorname{pr}_{1}(\widetilde{L})
$$

On the other hand, hypothesis (2) implies that

$$
\operatorname{pr}_{1}(\widetilde{L}) \subseteq \bar{B}^{2 n}(T)
$$

Therefore, applying Lemma 12, we have

$$
e(\widetilde{W}, \widetilde{L}) \leq e\left(\mathbb{R}^{2 n}, \operatorname{pr}_{1}(\widetilde{L})\right)<T
$$

Assume now by contradiction that $f$ was injective. This map is proper, since its domain is compact. Hence it follows from Lemma 10 that $f$ is a Lagrangian embedding. Since $\left(\mathbb{R}^{2 n}, \omega_{0}\right)$ and $(W, \omega)$ are geometrically bounded, the same holds for $(\widetilde{W}, \widetilde{\omega})$. Therefore, Theorem 11 implies that

$$
e(\widetilde{W}, \widetilde{L}) \geq A(\widetilde{W}, \widetilde{L})
$$

Combining this inequality with (19) and the next claim, we arrive at a contradiction.

Claim 2. We have

$$
A(\widetilde{W}, \widetilde{L}) \geq T
$$

Proof of Claim 2: Let $\widetilde{u} \in C^{\infty}(\mathbb{D}, \widetilde{W})$ be such that

$$
\widetilde{u}\left(S^{1}=\partial \mathbb{D}\right) \subseteq \widetilde{L}=f((\mathbb{R} / T \mathbb{Z}) \times L) .
$$

We show that

$$
\int_{\mathbb{D}} \widetilde{u}^{*} \widetilde{\omega} \in T \mathbb{Z}
$$

We define

$$
x:=\varphi^{-1} \circ \widetilde{u}: S^{1} \rightarrow \psi((\mathbb{R} / T \mathbb{Z}) \times L) \subseteq M .
$$

(Recall that $\varphi: M \rightarrow \widetilde{W}$ is the given embedding.) The equality $\widetilde{\omega}=d \widetilde{\lambda}$, Stokes' Theorem, and the hypothesis (3) imply that

$$
\int_{\mathbb{D}} \widetilde{u}^{*} \widetilde{\omega}=\int_{S^{1}}(\varphi \circ x)^{*} \widetilde{\lambda}=\int_{S^{1}} x^{*} \alpha .
$$


We define

$$
(z, y):=\psi^{-1} \circ x: S^{1} \rightarrow(\mathbb{R} / T \mathbb{Z}) \times L .
$$

This makes sense, since the restriction of $\psi$ to $(\mathbb{R} / T \mathbb{Z}) \times L$ is injective. (Here we use our assumption that $f$ is injective.) Since $x=\psi \circ(z, y)$, we have

$$
x^{*} \alpha=\alpha\left(R \circ \psi \circ(z, y) d z+d \psi_{z}(y) d y\right)=d z+\alpha d y=d z .
$$

Here we view $d z$ as a real-valued one-form on $S^{1}$. In the second equality we used that $\alpha(R) \equiv 1$ and that the Reeb flow $\psi$ preserves $\alpha$. In the last equality we used that $L$ is Legendrian, and hence $\left.\alpha\right|_{T L}=0$. It follows that

$$
\int_{S^{1}} x^{*} \alpha=\int_{S^{1}} d z=T \operatorname{deg}(z) .
$$

Using (22), this proves (20), i.e., Claim 2, and therefore Claim 1.

By Claim 1 there exist distinct points $\left(z_{i}, x_{i}\right) \in S^{1} \times L=(\mathbb{R} / T \mathbb{Z}) \times L$, $i=0,1$, such that

$$
f\left(z_{0}, x_{0}\right)=f\left(z_{1}, x_{1}\right) .
$$

Recalling the definition (14), our hypothesis that the period of every characteristic equals $T$, implies that the map $f\left(\cdot, x_{0}\right)$ is injective. It follows that $x_{0} \neq x_{1}$. Using (14]23), it follows that these two points lie on the same characteristic for $\alpha$. Hence $L$ intersects this characteristic at least twice. This proves Theorem 1.

Remark. The above proof relies on the sharp bound for the displacement energy of a closed Lagrangian submanifold due to Yu. Chekanov Ch2]. The same result was used by K. Mohnke [Mo] and later by K. Cieliebak and K. Mohnke [CM, Corollaries 1.4 and 1.5] to find Reeb chords. The construction of the closed Lagrangian submanifold in the proof of Theorem 1 is a variation on the construction in [Mo, $\mathbf{C M}$.

A new feature is that here the Reeb flow alone is used to produce a Lagrangian submanifold, whereas in [Mo, CM] both the Reeb flow and the Liouville flow are used. The new approach works because of the upper bound on the displacement energy of a compact subset of a ball given in Lemma 12.

\section{Proof of Proposition 3 (contact form)}

The proof of Proposition 3 is based on the following result. Let $(W, \rho, \lambda)$ be an exact Hamiltonian $S^{1}$-manifold, and $c \in \mathbb{R} \backslash\{0\}$. We denote by 
$X$ the vector field generated by $\rho$ and define

$$
H:=\lambda(X): W \rightarrow \mathbb{R}, \quad M:=H^{-1}(c) \subseteq W .
$$

We denote by

$$
\iota: M \rightarrow W
$$

the inclusion.

Proposition 13. The set $M$ is a hypersurface in $W, \alpha:=\iota^{*} \lambda$ is a contact form on $M$, and all characteristics of $\alpha$ are closed. Their periods are equal to $2 \pi c$ if the restriction of the action $\rho$ to $M$ is free.

Proof of Proposition 13. By hypothesis the form

$$
\omega:=d \lambda
$$

is nondegenerate, i.e., symplectic. We denote by $V$ the Liouville vector field on $W$ w.r.t. $\lambda$. This is the unique vector field satisfying

$$
\iota_{V} \omega=\lambda \text {. }
$$

We have

$$
\begin{aligned}
d H \cdot V & =\iota_{V} d \iota_{X} \lambda \\
& =\iota_{V} \mathcal{L}_{X} \lambda-\iota_{V} \iota_{X} d \lambda \\
& =0+\iota_{X} \iota_{V} \omega \\
& =\iota_{X} \lambda \\
& =H .
\end{aligned}
$$

Here in the second line we used Cartan's formula, and in the third line we used our hypothesis that $\lambda$ is $\rho$-invariant. It follows that $d H \cdot V \equiv$ $c \neq 0$ along $M=H^{-1}(c)$. Hence $c$ is a regular value for $H, M$ is a hypersurface in $W$, and the Liouville vector field $V$ is transverse to $M$. It follows that $\alpha:=\iota^{*} \lambda$ is a contact form on $M$. By the next claim its characteristics are closed.

Claim 1. The characteristics of $\alpha$ are the orbits of the restriction of $\rho$ to $M$.

Proof of Claim 1. It suffices to show that $X$ is $c$ times the Reeb vector field of $\alpha$. To see this, note that $X$ is tangent to $M$, since

$$
d H \cdot X=\iota_{X} d \iota_{X} \lambda=\iota_{X} \mathcal{L}_{X} \lambda-\iota_{X} \iota_{X} d \lambda=0-0 .
$$

By definition, we have

$$
\alpha(X)=\lambda(X) \equiv c \text { on } M=H^{-1}(c) .
$$


Finally,

$$
\iota_{X} d \alpha=\iota_{X} d \lambda=\mathcal{L}_{X} \lambda-d \iota_{X} \lambda=0-d H=0 \text { on } T M .
$$

It follows that $X$ equals $c$ times the Reeb vector field of $\alpha$. This proves Claim 1 .

Assume now that the restriction of $\rho$ to $M$ is free. Let $C$ be a characteristic for $\alpha$. We choose $x_{0} \in C$ and define

$$
\varphi: S^{1} \rightarrow C, \quad \varphi(z):=\rho\left(z, x_{0}\right) .
$$

This is a diffeomorphism, since the restriction of $\rho$ to $C$ is free. We denote by $\iota: C \rightarrow M$ the inclusion and by $\gamma$ the standard angular one-form on $S^{1}$, whose integral equals $2 \pi$. We have

$$
\varphi^{*} \iota^{*} \alpha=(\iota \circ \varphi)^{*} \alpha=(\lambda(X) \circ \varphi) \gamma=(H \circ \varphi) \gamma=c \gamma \text {. }
$$

Here in the second step we used the fact that $X$ generates the action $\rho$, and (24). It follows that

$$
\int_{C} \iota^{*} \alpha=\int_{S^{1}} \varphi^{*} \iota^{*} \alpha=2 \pi c .
$$

Here we equipped $C$ with the orientation induced by the standard orientation on $S^{1}$ and the map $\varphi$. This proves Proposition 13.

Proof of Proposition 3. We denote by $\rho_{0}$ the standard diagonal $S^{1}$ action on $\mathbb{R}^{2 n}=\mathbb{C}^{n}$, given by

$$
\rho_{0}(z) z_{0}:=z z_{0}=\left(z z_{0}^{1}, \ldots, z z_{0}^{n}\right) .
$$

By $\rho_{0} \times \rho$ we denote the product $S^{1}$-action on $\mathbb{R}^{2 n} \times W$. We define $H_{0}, H$ as in (4, 5). The triple

$$
(\widetilde{W}, \widetilde{\rho}, \widetilde{\lambda}):=\left(\left(\bar{B}^{2 n}(2 \pi c) \backslash\{0\}\right) \times W,\left.\left(\rho_{0} \times \rho\right)\right|_{\widetilde{W}}, \lambda_{0} \oplus \lambda\right)
$$

is an exact Hamiltonian $S^{1}$-action, and

$$
\widetilde{H}:=H_{0} \oplus H=\iota_{\widetilde{X}} \widetilde{\lambda}: \widetilde{W} \rightarrow \mathbb{R}
$$

where $\widetilde{X}$ denotes the vector field generated by $\widetilde{\rho}$. The set $M$ defined in (6), is given by

$$
M=\widetilde{H}^{-1}(c) \text {. }
$$

Since the restriction of $\rho_{0}$ to $\bar{B}^{2 n}(2 \pi c) \backslash\{0\}$ is free, the action $\widetilde{\rho}$ is free. Therefore, by Proposition $13 M$ is a hypersurface in $\widetilde{W}$, and $\alpha:=\iota^{*} \tilde{\lambda}$ is a contact form on $M$ all of whose characteristics are closed and of period $2 \pi c$. This proves Proposition 3 . 


\section{Proof of Theorem 6 (minimal action) and of Corollary 8 (presymplectic embedding)}

In this section we denote by

$$
B_{r}^{n}, \quad \bar{B}_{r}^{n}, \quad S_{r}^{n-1}
$$

the open and closed balls around 0 in $\mathbb{R}^{n}$ of radius $r$, and the sphere around 0 in $\mathbb{R}^{n}$ of radius $r$.

The proof of Theorem 6 is based on Lemma 12 (bound on displacement energy) and the following. Let $(M, \omega)$ be a symplectic manifold.

Theorem 14. Assume that $(M, \omega)$ is geometrically bounded. Let $N \subseteq$ $M$ be a closed, regular coisotropic submanifold. Then

$$
e(N) \geq A(N) \text {. }
$$

Proof. This is an immediate consequence of [Zi, Theorem 1.1].

Remark. This theorem generalizes Chekanov's Theorem 11.

In the proof of Theorem 6 we will also use the following lemma. Let $(M, \omega)$ be a presymplectic manifold. Recall that this means that $\omega$ is closed two-form on $M$, and corank $\omega_{x}:=\operatorname{dim}\left(T_{x} M\right)^{\omega}$ does not depend on $x \in M$. Let $N \subseteq M$ be a coisotropic submanifold. This means that for every $x \in N$ the space $\left(T_{x} N\right)^{\omega}$ is contained in $T_{x} N$. We denote by $\iota: N \rightarrow M$ the inclusion.

Remark 15. The form $\iota^{*} \omega$ is presymplectic. That its corank is constant, follows from Lemma 28 and Remark 30 in the appendix.

By Remark 15 the distribution $(T N)^{\omega}$ defines a foliation on $N$. We denote by $N_{\omega}$ the set of its leaves and define the action spectrum $S(N)=S(M, N)=S(M, \omega, N)$ and the minimal action (or area)

$$
A(N)=A(M, N)=A(M, \omega, N)
$$

of such a submanifold as in (8).9).

Lemma 16 (lift of coisotropic submanifold). Let $(M, \omega)$ and $\left(M^{\prime}, \omega^{\prime}\right)$ be presymplectic manifolds, $f: M^{\prime} \rightarrow M$ a surjective proper presymplectic $^{15}$ submersion, and $N \subseteq M$ a coisotropic submanifold. Then the following statements hold:

(i) The set $N^{\prime}:=f^{-1}(N)$ is a coisotropic submanifold of $M^{\prime}$.

\footnotetext{
${ }^{15}$ This means that $f^{*} \omega=\omega^{\prime}$.
} 
(ii)

$$
A(M, N) \leq A\left(M^{\prime}, N^{\prime}\right)
$$

(iii) Assume that $N$ is regular and, for all $x^{\prime}, y^{\prime} \in M^{\prime}$,

(26) $f\left(x^{\prime}\right)=f\left(y^{\prime}\right) \Rightarrow x^{\prime}$ and $y^{\prime}$ lie on the same isotropic leaf of $M^{\prime}$.

Then $N^{\prime}$ is regular.

Remark. In fact equality in (25) holds. However, this will not be used here.

In the proof of Lemma 16 we will use the following. By a presymplectic vector space we mean a vector space together with a skew-symmetric bilinear form.

Lemma 17. Let $(V, \omega)$ and $\left(V^{\prime}, \omega^{\prime}\right)$ be presymplectic vector spaces, $\Phi: V^{\prime} \rightarrow V$ a linear presymplectic map ${ }^{16}$, and $W \subseteq V$ a linear subspace. Then the following statements hold:

$$
\Phi^{-1}\left(W^{\omega}\right) \subseteq\left(\Phi^{-1}(W)\right)^{\omega^{\prime}} .
$$

(ii) If $\Phi$ is surjective then the inclusion " $\supseteq$ " in (27) holds.

Proof of Lemma 17. This follows from the definitions.

The proof of Lemma 16(iii) is based on the following. Let $M$ be a (smooth finite-dimensional) manifold and $\mathcal{F}$ a (smooth) foliation on $M$, i.e., a maximal atlas of foliation charts. We denote by $R^{\mathcal{F}}$ its leaf relation. This is the subset of $M \times M$ consisting of pairs of points lying in the same leaf. We call $\mathcal{F}$ regular iff there exists a manifold structure ${ }^{17}$ on the set of leaves $M / R^{\mathcal{F}}$, such that the canonical projection $\pi^{\mathcal{F}}$ : $M \rightarrow M / R^{\mathcal{F}}$ is a (smooth) submersion.

Lemma 18. Let $(M, \mathcal{F})$ and $\left(M^{\prime}, \mathcal{F}^{\prime}\right)$ be foliated manifolds, such that $\mathcal{F}$ is regular. Let $f: M^{\prime} \rightarrow M$ be a smooth surjective submersion such that

$$
x^{\prime} R^{\mathcal{F}^{\prime}} y^{\prime} \Longleftrightarrow f\left(x^{\prime}\right) R^{\mathcal{F}} f\left(y^{\prime}\right), \quad \forall x^{\prime}, y^{\prime} \in M^{\prime}
$$

Then $\mathcal{F}^{\prime}$ is regular.

\footnotetext{
${ }^{16}$ This means that $\Phi^{*} \omega=\omega^{\prime}$.

${ }^{17}$ The induced topology is by definition Hausdorff and second countable.
} 
Proof of Lemma 18. We define the map

$$
\varphi: M^{\prime} / R^{\mathcal{F}^{\prime}} \rightarrow M / R^{\mathcal{F}}, \quad \varphi\left(F^{\prime}\right):=\left[f\left(x^{\prime}\right)\right],
$$

where $x^{\prime} \in F^{\prime}$ is an arbitrary point. It follows from (28) that this map is well-defined and injective. Our hypothesis that $f$ is surjective implies that $\varphi$ is surjective, as well. By our assumption that $\mathcal{F}$ is regular there exists a manifold structure $\mathcal{A}$ on $M / R^{\mathcal{F}}$, for which the canonical projection $\pi^{\mathcal{F}}: M \rightarrow M / R^{\mathcal{F}}$ is a smooth submersion. Since $f$ is a smooth submersion and

$$
\pi^{\mathcal{F}^{\prime}}=\varphi^{-1} \circ \pi^{\mathcal{F}} \circ f
$$

the map $\pi^{\mathcal{F}^{\prime}}$ is a smooth submersion w.r.t. the pullback of $\mathcal{A}$ under $\varphi$. Hence $\mathcal{F}^{\prime}$ is regular. This proves Lemma 18 .

Proof of Lemma 16. (ii): Since $f$ is a submersion, $N^{\prime}$ is a submanifold of $M^{\prime}$. It follows from Lemma 17(iii) that it is coisotropic. This proves (ii).

To prove (iil (iii), we denote by $R^{N, \omega}$ the isotropic leaf relation on $N$. This is the subset of $N \times N$ consisting of pairs of points that lie in the same isotropic leaf of $N$.

Claim 1. (a) If $\left(x_{0}^{\prime}, x_{1}^{\prime}\right) \in R^{N^{\prime}, \omega^{\prime}}$ then $\left(f\left(x_{0}^{\prime}\right), f\left(x_{1}^{\prime}\right)\right) \in R^{N, \omega}$.

(b) If $x_{0}^{\prime}, x_{1}^{\prime} \in N^{\prime}$ are such that $\left(f\left(x_{0}^{\prime}\right), f\left(x_{1}^{\prime}\right)\right) \in R^{N, \omega}$ then

$$
N_{x_{0}^{\prime}}^{\prime} \cap f^{-1}\left(f\left(x_{1}^{\prime}\right)\right) \neq \emptyset \text {. }
$$

Here $N_{x_{0}^{\prime}}^{\prime}$ denotes the isotropic leaf of $N^{\prime}$ through $x_{0}^{\prime}$.

Proof of Claim 1. Let $x^{\prime} \in N^{\prime}$. Since $f$ is a submersion, we have

$$
T_{x^{\prime}} N^{\prime}=d f\left(x^{\prime}\right)^{-1}\left(T_{f\left(x^{\prime}\right)} N\right) \text {. }
$$

Using that $f$ is presymplectic, Lemma 17 therefore implies that

$$
\left(T_{x^{\prime}} N^{\prime}\right)^{\omega^{\prime}}=d f\left(x^{\prime}\right)^{-1}\left(T_{f\left(x^{\prime}\right)} N\right)^{\omega} .
$$

It follows that $f\left(N_{x^{\prime}}^{\prime}\right) \subseteq N_{f\left(x^{\prime}\right)}$. This proves (国).

Proof of $(\mathrm{b})$ : We choose a path $x \in C^{\infty}([0,1], N)$ that is tangent to $(T N)^{\omega}$ and satisfies $x(i)=f\left(x_{i}^{\prime}\right)$ for $i=0,1$. Since $f$ is a proper submersion, by Proposition 25 in the appendix there exists a path $x^{\prime} \in C^{\infty}\left([0,1], M^{\prime}\right)$ satisfying $x^{\prime}(0)=x_{0}^{\prime}$ and $f \circ x^{\prime}=x$. It follows that $x^{\prime}([0,1]) \subseteq N^{\prime}$. Since $\dot{x}(t) \in\left(T_{x(t)} N\right)^{\omega}$, equality (30) implies that $\dot{x}^{\prime}(t) \in\left(T_{x^{\prime}(t)} N^{\prime}\right)^{\omega^{\prime}}$, for every $t \in[0,1]$. It follows that $x^{\prime}(1) \in N_{x^{\prime}(0)}^{\prime}$. 
Since $x^{\prime}(0)=x_{0}^{\prime}$ and $f\left(x^{\prime}(1)\right)=x(1)=f\left(x_{1}^{\prime}\right)$, condition (29) follows. This proves (b) and completes the proof of Claim 1.

Proof of (iii): Let $u^{\prime} \in C^{\infty}\left(\mathbb{D}, M^{\prime}\right)$ be such that $u^{\prime}\left(S^{1}\right)$ is contained in some isotropic leaf of $N^{\prime}$. Claim 1(国) implies that $f \circ u^{\prime}\left(S^{1}\right)$ is contained in some isotropic leaf of $N$. Since $f$ is presymplectic, we have

$$
\int_{\mathbb{D}} u^{\prime *} \omega^{\prime}=\int_{\mathbb{D}}\left(f \circ u^{\prime}\right)^{*} \omega
$$

It follows that $S\left(M^{\prime}, N^{\prime}\right) \subseteq S(M, N)$, and therefore,

$$
A\left(M^{\prime}, N^{\prime}\right) \geq A(M, N) \text {. }
$$

This proves (iil).

Proof of (iii): By Claim 1(国) the implication " $\Rightarrow$ " in condition (28) with $M, M^{\prime}$ replaced by $N, N^{\prime}$, and $\mathcal{F}=\mathcal{F}^{N, \omega}, \mathcal{F}^{\prime}=\mathcal{F}^{N^{\prime}, \omega^{\prime}}$, is satisfied. Here $\mathcal{F}^{N, \omega}$ denotes the isotropic foliation on $N$ w.r.t. $\omega$.

To see the opposite implication, let $x_{0}^{\prime}, x_{1}^{\prime} \in N^{\prime}$ be such that the relation $f\left(x_{0}^{\prime}\right) R^{N, \omega} f\left(x_{1}^{\prime}\right)$ holds. By Claim [1(b) there exists $y_{1}^{\prime} \in N_{x_{0}^{\prime}}^{\prime} \cap$ $f^{-1}\left(f\left(x_{1}^{\prime}\right)\right)$. Since $f\left(x_{1}^{\prime}\right)=f\left(y_{1}^{\prime}\right)$, our hypothesis (26) implies that

$$
\left(x_{1}^{\prime}, y_{1}^{\prime}\right) \in R^{M^{\prime}, \omega^{\prime}} \cap\left(N^{\prime} \times N^{\prime}\right) \subseteq R^{N^{\prime}, \omega^{\prime}} .
$$

Since $\left(x_{0}^{\prime}, y_{1}^{\prime}\right) \in R^{N^{\prime}, \omega^{\prime}}$, it follows that $\left(x_{0}^{\prime}, x_{1}^{\prime}\right) \in R^{N^{\prime}, \omega^{\prime}}$. This shows the implication " $\Leftarrow$ " in (28) with $M, M^{\prime}$ replaced by $N, N^{\prime}$, and $\mathcal{F}=\mathcal{F}^{N, \omega}$, $\mathcal{F}^{\prime}=\mathcal{F}^{N^{\prime}, \omega^{\prime}}$. Hence (28) is satisfied. Therefore, applying Lemma 18, it follows that $N^{\prime}$ is regular. This proves (iii) and completes the proof of Lemma 16.

In the proof of Theorem 6 we will also use the following lemma.

Lemma 19. Let $(M, \omega)$ be a presymplectic manifold, $M^{\prime}$ a coisotropic submanifold of $M$ and $M^{\prime \prime}$ a coisotropic submanifold of $M^{\prime}$. Then the following holds.

(i) $M^{\prime \prime}$ is a coisotropic submanifold of $M$.

(ii) If $M$ strongly smoothly deformation retracts onto $M^{\prime}$ then

$$
A\left(M^{\prime}, M^{\prime \prime}\right) \leq A\left(M, M^{\prime \prime}\right) .
$$

Remarks 20. (i) That $M$ strongly smoothly deformation retracts onto $M^{\prime}$ means that there exists a smooth map $h:[0,1] \times M \rightarrow M$ such that

$$
h(0, \cdot)=\mathrm{id}, \quad h(\{1\} \times M) \subseteq M^{\prime}, \quad h(t, x)=x, \forall t \in[0,1], x \in M^{\prime} .
$$


(ii) The inequality " $\geq$ " in (31) is true without the retraction condition. However, this will not be used here.

In the proof of Lemma 19 we will use the following lemma.

Lemma 21. Let $(V, \omega)$ be a finite-dimensional presymplectic vector space, $V^{\prime}$ a coisotropic subspace of $(V, \omega)$, and $V^{\prime \prime}$ a coisotropic subspace of $\left(V^{\prime}, \omega^{\prime}:=\left.\omega\right|_{V^{\prime} \times V^{\prime}}\right)$. Then $V^{\prime \prime}$ is a coisotropic subspace of $(V, \omega)$.

Proof of Lemma 21. This follows from Lemma 28 in the appendix.

Proof of Lemma 19. (i): This follows from Lemma 21.

We prove (ii). It suffices to show that

$$
S\left(M, M^{\prime \prime}\right) \subseteq S\left(M^{\prime}, M^{\prime \prime}\right) .
$$

Let $u \in C^{\infty}(\mathbb{D}, M)$ be such that $u\left(S^{1}\right)$ is contained in some isotropic leaf of $M^{\prime \prime}$. We choose a map $h$ as in Remark 20(i). We denote $h_{t}:=$ $h(t, \cdot)$ and define

$$
f:[0,1] \times \mathbb{D} \rightarrow M, \quad f(t, z):=h_{t} \circ u(z) .
$$

Using that $d \omega=0$ and Stokes' theorem ${ }^{18}$, we have

$$
\begin{aligned}
0 & =\int_{[0,1] \times \mathbb{D}} d f^{*} \omega \\
& =\int_{\partial([0,1] \times \mathbb{D})} f^{*} \omega \\
& =\int_{\mathbb{D}}\left(h_{1} \circ u\right)^{*} \omega-\int_{\mathbb{D}}\left(h_{0} \circ u\right)^{*} \omega+\int_{[0,1] \times S^{1}} f^{*} \omega \\
& =\int_{\mathbb{D}}\left(h_{1} \circ u\right)^{*} \omega-\int_{\mathbb{D}} u^{*} \omega+0 .
\end{aligned}
$$

Here in the last equality we used the fact $h_{0}=$ id and that $u\left(S^{1}\right) \subseteq$ $M^{\prime \prime} \subseteq M^{\prime}$, and therefore $\left.h_{t} \circ u\right|_{S^{1}}$ is constant in $t$. Since $h_{1}(M)=$ $h(\{1\} \times M) \subseteq M^{\prime}$, the map $h_{1} \circ u$ takes values in $M^{\prime}$. It is of the sort occurring in the definition of $S\left(M^{\prime}, M^{\prime \prime}\right)$. Hence (33) implies (32). This proves (iii) and completes the proof of Lemma 19.

In the proof of Theorem 6 we will also use the following lemma.

\footnotetext{
${ }^{18}$ We use a version of this result that allows the manifold to have corners. See e.g. [Le, Theorem 16.25].
} 
Lemma 22. Let $(M, \omega)$ and $\left(M^{\prime}, \omega^{\prime}\right)$ be presymplectic manifolds, $N \subseteq$ $M \times M^{\prime}$ a coisotropic submanifold, and $x \in M$, such that $\operatorname{dim} M>2$ and $N \cap\left(\{x\} \times M^{\prime}\right)=\emptyset$. Then

$$
A\left((M \backslash\{x\}) \times M^{\prime}, N\right) \leq A\left(M \times M^{\prime}, N\right) .
$$

Remark. In fact equality in (34) holds. However, this will not be used here.

Proof of Lemma 20. It suffices to prove that

$$
S\left(M \times M^{\prime}, N\right) \subseteq S\left((M \backslash\{x\}) \times M^{\prime}, N\right) .
$$

Let

$$
\widetilde{u}=\left(u, u^{\prime}\right) \in C^{\infty}\left(\mathbb{D}, M \times M^{\prime}\right)
$$

be a map that sends $S^{1}$ to some isotropic leaf of $N$. Since $\operatorname{dim} M>2$, by Sard's Theorem $M \backslash u\left(B_{1}^{2}\right)$ is dense in $M$. Hence an argument in a chart shows that there exists a smooth map $h:[0,1] \times M \rightarrow M$, such that

$$
\begin{gathered}
h(0, \cdot)=\mathrm{id}, \quad x \notin h\left(\{1\} \times u\left(B_{1}^{2}\right)\right), \\
h(t, \cdot)=\text { id in some neighbourhood of } \operatorname{pr}_{1}(N) \subseteq M .
\end{gathered}
$$

Here we denoted by $\mathrm{pr}_{1}: M \times M^{\prime} \rightarrow M$ the canonical projection, and we used the hypothesis that $N \cap\left(\{x\} \times M^{\prime}\right)=\emptyset$. We denote $h_{t}:=h(t, \cdot)$ and define

$$
f:[0,1] \times \mathbb{D} \rightarrow M, \quad f(t, z):=h_{t} \circ u(z) .
$$

We have, as in (33),

$$
\int_{\mathbb{D}}\left(h_{1} \circ u\right)^{*} \omega=\int_{\mathbb{D}} u^{*} \omega .
$$

Here we used the facts $h_{0}=\mathrm{id}, u\left(S^{1}\right)=\operatorname{pr}_{1} \circ \widetilde{u}\left(S^{1}\right) \subseteq \operatorname{pr}_{1}(N)$, and $h_{t}=\mathrm{id}$ in a neighbourhood of $\operatorname{pr}_{1}(N)$. It follows that

$$
\int_{\mathbb{D}}\left(h_{1} \circ u, u^{\prime}\right)^{*} \widetilde{\omega}=\int_{\mathbb{D}} \widetilde{u}^{*} \widetilde{\omega} .
$$

Since $x \notin h_{1}\left(u\left(B_{1}^{2}\right)\right)$, the map

$$
\left(h_{1} \circ u, u^{\prime}\right): \mathbb{D} \rightarrow(M \backslash\{0\}) \times M^{\prime}
$$

is of the sort occurring in the definition of $S\left((M \backslash\{x\}) \times M^{\prime}, N\right)$. Hence (36) implies (35). This proves Lemma 22.

In the proof of Theorem 6 we will also use the following. 
Lemma 23. Let $(M, \omega)$ be a connected symplectic manifold and $N \subseteq$ $M$ coisotropic submanifold. Then

$$
S(N)+\left\{\int_{S^{2}} u^{*} \omega \mid u \in C^{\infty}\left(S^{2}, M\right)\right\} \subseteq S(N) .
$$

Proof of Lemma 23. Let $u \in C^{\infty}(\mathbb{D}, M)$ be such that $u\left(S^{1}\right)$ is contained in some isotropic leaf of $N$, and $v \in C^{\infty}\left(S^{2}, M\right)$. We choose a point $z_{0} \in S^{2}$.

Claim 1. There exist maps $\widetilde{u} \in C^{\infty}\left(\mathbb{D} \backslash B_{\frac{1}{2}}^{2}, M\right)$ and $\widetilde{v} \in C^{\infty}\left(\bar{B}_{\frac{1}{3}}^{2}, M\right)$ such that

$$
\int_{\mathbb{D} \backslash B_{\frac{1}{2}}^{2}} \widetilde{u}^{*} \omega=\int_{\mathbb{D}} u^{*} \omega, \quad \int_{\bar{B}_{\frac{1}{3}}^{2}} \widetilde{v}^{*} \omega=\int_{S^{2}} v^{*} \omega
$$

$\widetilde{u}=u$ in some neighbourhood of $S^{1}, \widetilde{u} \equiv u(0)$ in some neighbourhood of $S_{\frac{1}{2}}^{1}$, and $\widetilde{v} \equiv v\left(z_{0}\right)$ in some neighbourhood of $S_{\frac{1}{3}}^{1}$.

Proof of Claim 1 . We choose a map $f \in C^{\infty}\left(\mathbb{D} \backslash B_{\frac{1}{2}}^{2}, \mathbb{D}\right)$ that restricts to an orientation preserving diffeomorphism from $\mathbb{D} \backslash \bar{B}_{\frac{3}{4}}^{2}$ to $\mathbb{D} \backslash\{0\}$, equals identity in a neighbourhood of $S^{1}$, and sends $\bar{B}_{\frac{3}{4}}^{2} \backslash B_{\frac{1}{2}}^{2}$ to 0 . We define

$$
\widetilde{u}:=u \circ f: \mathbb{D} \backslash B_{\frac{1}{2}}^{2} \rightarrow M
$$

This map has the required properties.

To construct $\widetilde{v}$, we choose a map $g \in C^{\infty}\left(\bar{B}_{\frac{1}{3}}^{2}, S^{2}\right)$ that restricts to an orientation preserving diffeomorphism from $B_{\frac{1}{4}}^{2}$ to $S^{2} \backslash\left\{z_{0}\right\}$ and sends $\bar{B}_{\frac{1}{3}}^{2} \backslash B_{\frac{1}{4}}^{2}$ to $z_{0}$. The map $\widetilde{v}:=v \circ g$ has the required properties. This proves Claim 1 .

We choose $\widetilde{u}, \widetilde{v}$ as in this claim. Since $M$ is connected, there exists a path $x \in C^{\infty}\left(\left[\frac{1}{3}, \frac{1}{2}\right], M\right)$, such that $x(0)=v\left(z_{0}\right)$ and $x(1)=u(0)$. We may modify $x$, such that it is constant in some neighbourhoods of $\frac{1}{3}$ and $\frac{1}{2}$. We define

$$
w(z):= \begin{cases}\widetilde{v}(z), & \text { if } z \in \bar{B}_{\frac{1}{3}}^{2}, \\ x(|z|), & \text { if } z \in B_{\frac{1}{2}}^{2} \backslash \bar{B}_{\frac{1}{3}}^{2}, \\ \widetilde{u}(z), & \text { if } z \in \mathbb{D} \backslash B_{\frac{1}{2}}^{2} .\end{cases}
$$


This map is smooth. It follows from (38) that

$$
\int_{\mathbb{D}} w^{*} \omega=\int_{S^{2}} v^{*} \omega+0+\int_{\mathbb{D}} u^{*} \omega .
$$

Since $w=u$ in some neighbourhood of $S^{1}$, the image $w\left(S^{1}\right)$ is contained in some isotropic leaf of $N$. It follows that $\int_{\mathbb{D}} w^{*} \omega \in S(N)$. Combining this with (39), the inclusion (37) follows. This proves Lemma 23.

In the proof of Theorem [6] we will also use the following.

Remark 24. Let $(M, \omega)$ be a symplectic manifold and $N \subseteq M a$ coisotropic submanifold. Then

$$
S(N)=-S(N)=\{-a \mid a \in S(N)\} .
$$

This follows from the fact that for every $u \in C^{\infty}(\mathbb{D}, M)$ we have

$$
\int_{\mathbb{D}} \bar{u}^{*} \omega=-\int_{\mathbb{D}} u^{*} \omega
$$

where $\bar{u}(z):=u(\bar{z})$, for every $z \in \mathbb{D} \subseteq \mathbb{C}$.

Proof of Theorem [6. We denote by

$$
\pi: S^{2 n+1} \times M \rightarrow \mathbb{C P}^{n} \times M
$$

the canonical projection, by $\iota: S^{2 n+1} \rightarrow \mathbb{R}^{2 n}$ the inclusion, and by $\omega_{0}$ the standard symplectic form on $\mathbb{R}^{2 n}$. We equip $S^{2 n+1} \times M$ with the presymplectic form $\iota^{*} \omega_{0} \oplus \omega$. It follows from Lemma 16)(iii) that $N^{\prime}=\pi^{-1}(N)$ is a coisotropic submanifold of $S^{2 n+1} \times M$, and

$$
A\left(\mathbb{C P}^{n} \times M, N\right) \leq A\left(S^{2 n+1} \times M, N^{\prime}\right) .
$$

Since $\pi$ is proper and $N$ is compact, $N^{\prime}$ is compact. The manifold $\left(\mathbb{R}^{2 n+2} \backslash\{0\}\right) \times M$ strongly smoothly deformation retracts onto $S^{2 n+1} \times$ $M$. Hence by Lemma 19(ii), we have

$$
A\left(S^{2 n+1} \times M, N^{\prime}\right) \leq A\left(\left(\mathbb{R}^{2 n+2} \backslash\{0\}\right) \times M, N^{\prime}\right) .
$$

Since $n \geq 1$, by Lemma 22 we have

$$
A\left(\left(\mathbb{R}^{2 n+2} \backslash\{0\}\right) \times M, N^{\prime}\right) \leq A\left(\mathbb{R}^{2 n+2} \times M, N^{\prime}\right) .
$$

The symplectic manifold $\mathbb{R}^{2 n+2}$ is geometrically bounded. Using our hypothesis that $M$ is geometrically bounded, it follows that $\mathbb{R}^{2 n+2} \times M$ has the same property. Since by hypothesis $N$ is regular, by Lemma 16(iii) with $f=\pi$ the same holds for $N^{\prime}$. (Condition (26) with $M^{\prime}:=$ $S^{2 n+1} \times M$ is satisfied, since

$$
R^{S^{2 n+1} \times M}=\left\{((x, y),(z x, y)) \mid(x, y) \in S^{2 n+1} \times M, z \in S^{1}\right\},
$$


where we consider $S^{2 n+1}$ as a subset of $\mathbb{C}^{n+1}$ and $S^{1} \subseteq \mathbb{C}$.) Hence applying Theorem [14, we obtain

$$
A\left(\mathbb{R}^{2 n+2} \times M, N^{\prime}\right) \leq e\left(\mathbb{R}^{2 n+2} \times M, N^{\prime}\right) .
$$

We denote by $\operatorname{pr}_{1}: S^{2 n+1} \times M \rightarrow S^{2 n+1}$ the projection onto the first factor. We have

$$
\begin{aligned}
e\left(\mathbb{R}^{2 n+2} \times M, N^{\prime}\right) & \leq e\left(\mathbb{R}^{2 n+2} \times M, \operatorname{pr}_{1}\left(N^{\prime}\right) \times M\right) \\
& \leq e\left(\mathbb{R}^{2 n+2}, \operatorname{pr}_{1}\left(N^{\prime}\right)\right) .
\end{aligned}
$$

Our hypothesis $\operatorname{dim} M<2 n$ implies that $\operatorname{dim} N^{\prime}=\operatorname{dim} N+1 \leq 2 n$. Hence the restriction $\left.\mathrm{pr}_{1}\right|_{N^{\prime}}: N^{\prime} \rightarrow S^{2 n+1}$ is not submersive at any point, and therefore the set of its regular values is the complement of its image. Hence by Sard's Theorem $\operatorname{pr}_{1}\left(N^{\prime}\right) \neq S^{2 n+1}$. Therefore by Lemma 12 we have

$$
e\left(\mathbb{R}^{2 n+2}, \operatorname{pr}_{1}\left(N^{\prime}\right)\right)<\pi .
$$

Combining this with (40,44), it follows that

$$
A(N)=A\left(\mathbb{C P}^{n} \times M, N\right)<\pi
$$

Hence there exists $a \in S(N) \cap(0, \pi)$. If $a \leq \frac{\pi}{2}$ then it follows that $A(N) \leq \frac{\pi}{2}$, as claimed. Otherwise $-a+\pi<\frac{\pi}{2}$. By Remark 24 we have $-a \in S(N)$. Since there exists $u \in C^{\infty}\left(S^{2}, \mathbb{C P}^{n}\right)$, such that $\int_{S^{2}} u^{*} \omega_{\mathrm{FS}}=\pi$, Lemma 23 implies that $-a+\pi \in S(N)$. Since $-a+\pi<$ $\frac{\pi}{2}$, it follows that $A(N)<\frac{\pi}{2}$. Hence in every case we have $A(N) \leq \frac{\pi}{2}$. This proves Theorem 6 .

Proof of Corollary 8 . We denote

$$
\widetilde{M}:=\mathbb{C P}^{n} \times M, \quad \widetilde{\omega}:=\omega_{\mathrm{FS}} \oplus \omega .
$$

Assume by contradiction that there exists a presymplectic embedding $\varphi: M^{\prime} \rightarrow \widetilde{M}$. We denote $N:=\varphi\left(M^{\prime}\right)$. It follows from our hypothesis (11) and Lemma 28 in the appendix that $N$ is coisotropic. It is regular, since $M^{\prime}$ is regular.

Claim 2. We have

$$
A(\widetilde{M}, N) \geq \pi
$$

Proof of Claim 2. It follows from our hypothesis (10) that

$$
\int_{S^{2}} \widetilde{w}^{*} \widetilde{w} \in \pi \mathbb{Z}, \quad \forall \widetilde{w} \in C^{\infty}\left(S^{2}, \widetilde{M}\right) .
$$

Let $\widetilde{u} \in C^{\infty}(\mathbb{D}, \widetilde{M})$ be such that $\widetilde{u}\left(S^{1}\right)$ is contained in some isotropic leaf $F$ of $N$. We choose a map $f \in C^{\infty}(\mathbb{D}, \mathbb{D})$ that restricts to an 
orientation preserving diffeomorphism from $B_{\frac{1}{2}}^{2}$ to $B_{1}^{2}$ and satisfies $f(z)=z /|z|$ on $\mathbb{D} \backslash B_{\frac{1}{2}}^{2}$.

The pre-image $\varphi^{-1}(F)$ is an isotropic leaf of $M^{\prime}$. By hypothesis it is simply-connected. Hence the same holds for $F$. It follows that there exists a map $\widetilde{v} \in C^{\infty}(\mathbb{D}, F)$ satisfying $\widetilde{v}=\widetilde{u}$ on $S^{1}$. Modifying $\widetilde{v}$, we may assume that $\widetilde{v}(z)=\widetilde{v}(z /|z|)$ for every $z \in \mathbb{D} \backslash B_{\frac{1}{2}}^{2}$.

We denote by $\overline{\mathbb{D}}$ the disk with the reversed orientation and by $\mathbb{D} \# \overline{\mathbb{D}}$ the smooth oriented manifold obtained by concatenating the two disks along their boundary. We define $\widetilde{w}: \mathbb{D} \# \overline{\mathbb{D}} \rightarrow \mathbb{C P}^{n} \times M$ to be the concatenation of $\widetilde{u} \circ f$ and $\widetilde{v}$. This is a smooth map. It follows that

$$
\int_{\mathbb{D} \# \overline{\mathbb{D}}} \widetilde{w}^{*} \widetilde{\omega}=\int_{\mathbb{D}}(\widetilde{u} \circ f)^{*} \widetilde{\omega}-\int_{\mathbb{D}} \widetilde{v}^{*} \widetilde{\omega}=\int_{\mathbb{D}} \widetilde{u}^{*} \widetilde{\omega}-0 .
$$

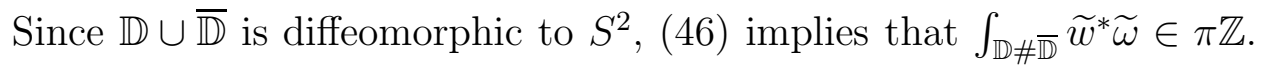
Combining this with (47), inequality (45) follows. This proves Claim 2 .

This claim and the hypothesis $\operatorname{dim} M^{\prime}<2 n$ contradict Theorem 6, Hence the presymplectic embedding $\varphi: M^{\prime} \rightarrow \widetilde{M}$ does not exist. This proves Corollary 8 ,

\section{Appendix A. Lifting paths}

The following result was used in the proof of Lemma 16. Let $M^{\prime}, M$ be smooth manifolds, $f: M^{\prime} \rightarrow M$ a smooth proper submersion, $p^{\prime} \in M^{\prime}$, and $x \in C^{\infty}([0,1], M)$.

Proposition 25 (lifting a path). If $f\left(p^{\prime}\right)=x(0)$ then there exists a path $x^{\prime} \in C^{\infty}\left([0,1], M^{\prime}\right)$, such that

$$
f \circ x^{\prime}=x, \quad x^{\prime}(0)=p^{\prime} .
$$

The proof of this lemma is based on the following.

Lemma 26 (locally lifting a path). Let $t_{0} \in[0,1]$ and $H \subseteq T M^{\prime}$ be a (smooth) subbundle that is complementary to ker $d f$, i.e., satisfies $T M^{\prime}=H \oplus \operatorname{ker} d f$.

(i) (local existence) If $f\left(p^{\prime}\right)=x\left(t_{0}\right)$ then there exists a (relatively) open neighbourhood $V$ of $t_{0}$ in $[0,1]$ and a path $x^{\prime} \in C^{\infty}\left(V, M^{\prime}\right)$, satisfying

$$
\begin{gathered}
\dot{x}^{\prime}(t) \in H_{x^{\prime}(t)}, \quad f \circ x^{\prime}(t)=x(t), \quad \forall t \in V, \\
x^{\prime}\left(t_{0}\right)=p^{\prime} .
\end{gathered}
$$


(ii) (local uniqueness) If $V_{0}, V_{1}$ are open neighbourhoods of $t_{0}$ in $[0,1]$ and $x_{0}^{\prime}, x_{1}^{\prime} \in C^{\infty}\left([0,1], M^{\prime}\right)$ are paths, satisfying $(48,49)$ then there exists an open neighbourhood $V \subseteq V_{0} \cap V_{1}$ of $t_{0}$ in $[0,1]$, such that $x_{0}^{\prime}=x_{1}^{\prime}$ on $V$.

Remark 27 (global uniqueness). For $i=0,1$ let $t_{i} \in[0,1]$ and $x_{i}^{\prime} \in$ $C^{\infty}\left(\left[0, t_{i}\right], M^{\prime}\right)$ be a path satisfying (48) and $x_{i}^{\prime}(0)=p^{\prime}$. Then $x_{0}^{\prime}=x_{1}^{\prime}$ on $\left[0, \min \left\{t_{0}, t_{1}\right\}\right]$. This follows from Lemma [26)(ii).

Proof of Lemma 26. By using a chart in $M$ we may assume w.l.o.g. that $M=\mathbb{R}^{n}$. Using the Implicit Function Theorem and our hypothesis that $f$ is a smooth submersion, we may further assume w.l.o.g. that $M^{\prime}=\mathbb{R}^{m} \times \mathbb{R}^{n}$ and $f=\operatorname{pr}_{2}: \mathbb{R}^{m} \times \mathbb{R}^{n} \rightarrow \mathbb{R}^{n}$, the canonical projection.

For $t \in[0,1]$ and $y \in \mathbb{R}^{m}$ we define $X_{t}(y) \in \mathbb{R}^{m}$ to be the unique vector, such that

$$
\left(X_{t}(y), \dot{x}(t)\right) \in H_{(y, x(t))} .
$$

This vector exists and is unique, since $H$ is complementary to ker $d f=$ ker $\mathrm{pr}_{2}$. The family $\left(X_{t}\right)_{t \in[0,1]}$ is a smooth time-dependent vector field on $\mathbb{R}^{m}$. We write $p^{\prime}=\left(y_{0}, x\left(t_{0}\right)\right)$.

We prove (i). By the Picard-Lindelöf theorem there exist an open neighbourhood $V$ of $t_{0}$ in $[0,1]$ and a smooth solution $y \in C^{\infty}\left(V, \mathbb{R}^{m}\right)$ of the ordinary differential equation

$$
\dot{y}=X_{t} \circ y, \quad y\left(t_{0}\right)=y_{0} .
$$

Using (50), the path $x^{\prime}:=(y, x):[0,1] \rightarrow M^{\prime}=\mathbb{R}^{m} \times \mathbb{R}^{n}$ satisfies (48, 49). This proves (ii).

Statement (ii) follows from a similar argument. This proves Lemma 26.

Proof of Proposition 25. We choose a subbundle $H \subseteq T M^{\prime}$ that is complementary to ker $d f$. (We may define $H$ to be the normal bundle of ker $d f$ with respect to some Riemannian metric.) We define

$$
\begin{aligned}
& y^{\prime}:=\bigcup\left\{x^{\prime} \mid t_{1} \in[0,1], x^{\prime} \in C^{\infty}\left(\left[0, t_{1}\right], M^{\prime}\right):\right. \\
&\text { (48) with } \left.V=\left[0, t_{1}\right], x^{\prime}(0)=p^{\prime}\right\} \subseteq[0,1] \times M^{\prime} .
\end{aligned}
$$

It follows from Remark 27 that there exists $t_{0} \in[0,1]$ such that $y^{\prime}$ is a smooth map from $\left[0, t_{0}\right)$ or $\left[0, t_{0}\right]$ to $M^{\prime}$. Proposition 25] is a consequence of the following claim. 
Claim 1. The domain of $y^{\prime}$ is $[0,1]$.

Proof of Claim 1; We define

$$
X:=\left\{\left(x^{\prime}, v\right) \mid v \in T_{f\left(x^{\prime}\right)} M\right\}, \quad \Phi: X \rightarrow H, \Phi_{x^{\prime}} v:=\Phi\left(x^{\prime}, v\right):=v^{\prime},
$$

where $v^{\prime} \in H_{x^{\prime}}$ is the unique vector satisfying $d f\left(x^{\prime}\right) v^{\prime}=v$. Since $d f\left(x^{\prime}\right)$ is surjective and $T_{x^{\prime}} M^{\prime}=H_{x^{\prime}} \oplus \operatorname{ker} d f\left(x^{\prime}\right)$, this vector exists and is unique, hence $\Phi$ is well-defined. This map is smooth, since $H$ is smooth. We choose Riemannian metrics $g$ on $M$ and $g^{\prime}$ on $M^{\prime}$. Since $f$ is proper, the pre-image $K^{\prime}:=f^{-1}(x([0,1])) \subseteq M^{\prime}$ is compact. Therefore,

$$
C:=\sup \left\{\left|\Phi_{x^{\prime}}\right| \mid x^{\prime} \in K^{\prime}\right\}<\infty .
$$

Here $\left|\Phi_{x^{\prime}}\right|$ denotes the operator norm of the linear map $\Phi_{x^{\prime}}: T_{f\left(x^{\prime}\right)} M \rightarrow$ $T_{x^{\prime}} M^{\prime}$ w.r.t. the norms induced by $g$ and $g^{\prime}$.

Let $t \in\left[0, t_{0}\right)$. By (48) we have $\dot{y}^{\prime}(t) \in H_{y^{\prime}(t)}$ and $d f\left(y^{\prime}(t)\right) \dot{y}^{\prime}(t)=$ $\dot{x}(t)$, and therefore

$$
\dot{y}^{\prime}(t)=\Phi_{y^{\prime}(t)} \dot{x}(t) .
$$

Since $y^{\prime}\left(\left[0, t_{0}\right)\right) \subseteq K^{\prime}$, it follows that

$$
\left|\dot{y}^{\prime}(t)\right| \leq C|\dot{x}(t)| \leq C \max _{t \in[0,1]}|\dot{x}(t)| .
$$

It follows that $y^{\prime}(t)$ converges to some point $y_{0}^{\prime}$, as $t \uparrow t_{0}$.

Assume now by contradiction that the domain of $y^{\prime}$ is not equal to $[0,1]$. We choose $V, x^{\prime}$ as in Lemma 26)(ii), with $p^{\prime}$ replaced by $y_{0}^{\prime}$. Concatenating $y^{\prime}$ with $x^{\prime}$, we obtain a solution $z^{\prime}$ of (48) with $V$ replaced by an interval that strictly contains the domain of $y^{\prime}$, such that $z^{\prime}(0)=$ $p^{\prime}$. (Here we use Lemma 26(ii), which ensures that $x^{\prime}$ and $y^{\prime}$ agree on the intersection of $V$ with the domain of $y^{\prime}$, if we shrink $V$.) By (51) we have $z^{\prime} \subseteq y^{\prime}$. This is a contradiction. It follows that the domain of $y^{\prime}$ is equal to $[0,1]$. This proves Claim 1 and completes the proof of Proposition 25.

\section{Appendix B. Coisotropic subspaces of presymplectic vector spaces}

The following lemma was used in the proof of Lemma 21, Let $(V, \omega)$ be a finite-dimensional presymplectic vector space and $W \subseteq V$ a linear subspace. We denote by $i: W \rightarrow V$ the inclusion, and by

$$
W^{\omega}:=\{v \in V \mid \omega(v, w)=0, \forall w \in W\}
$$

the presymplectic complement of $W$ in $V$. 
Lemma 28. The subspace $W$ is coisotropic iff

$$
\operatorname{dim} W+\operatorname{dim} W^{i^{*} \omega} \geq \operatorname{dim} V+\operatorname{dim} V^{\omega}{ }^{19}
$$

The proof of this lemma is based on the following.

Lemma 29. We have

$$
\operatorname{dim} W+\operatorname{dim} W^{\omega}=\operatorname{dim} V+\operatorname{dim}\left(V^{\omega} \cap W\right) .
$$

Remark 30. Since $W^{i^{*} \omega} \subseteq W^{\omega}$, Lemma 29 implies that inequality " $\leq$ " in (52) holds, for every linear subspace $W$.

Proof of Lemma 29. We define the linear map

$$
b_{\omega}: V \rightarrow V^{*}, \quad b_{\omega} v:=\omega(v, \cdot) .
$$

Then $W^{\omega}=\operatorname{ker}\left(i^{*} b_{\omega}\right)$, and therefore,

$$
\operatorname{dimim}\left(i^{*} b_{\omega}\right)+\operatorname{dim} W^{\omega}=\operatorname{dim} V .
$$

Consider the canonical isomorphism $\iota: V \rightarrow V^{* *}, \iota(v)(\varphi):=\varphi(v)$. A direct calculation shows that $\left(b_{\omega}\right)^{*} \iota=-b_{\omega}$. It follows that $\left(b_{\omega} i\right)^{*} \iota=$ $-i^{*} b_{\omega}$, and therefore

$$
\operatorname{dimim}\left(b_{\omega} i\right)=\operatorname{dimim}\left(b_{\omega} i\right)^{*}=\operatorname{dimim}\left(\left(b_{\omega} i\right)^{*} \iota\right)=\operatorname{dimim}\left(i^{*} b_{\omega}\right) .
$$

Combining this with (54), we obtain

$$
\begin{aligned}
\operatorname{dim} W+\operatorname{dim} W^{\omega} & =\operatorname{dim} \operatorname{ker}\left(b_{\omega} i\right)+\operatorname{dim} \operatorname{im}\left(b_{\omega} i\right)+\operatorname{dim} W^{\omega} \\
& =\operatorname{dim} \operatorname{ker}\left(b_{\omega} i\right)+\operatorname{dim} V .
\end{aligned}
$$

Since $\operatorname{ker}\left(b_{\omega} i\right)=V^{\omega} \cap W$, equality (53) follows. This proves Lemma 29 .

Proof of Lemma 28. We prove " $\Rightarrow$ ". Assume that $W$ is coisotropic. Then $W^{\omega} \subseteq W^{i^{*} \omega}$ and therefore, using Lemma 29, we have

$$
\begin{aligned}
\operatorname{dim} W+\operatorname{dim} W^{i^{*} \omega} & \geq \operatorname{dim} W+\operatorname{dim} W^{\omega} \\
& =\operatorname{dim} V+\operatorname{dim}\left(V^{\omega} \cap W\right) .
\end{aligned}
$$

Since $V^{\omega} \subseteq W^{\omega} \subseteq W$, inequality (52) follows. This proves " $\Rightarrow$ ".

\footnotetext{
${ }^{19}$ By definition, $W^{i^{*} \omega}$ is the presymplectic complement of $W$ inside $W$.
} 
To prove the opposite implication, assume that (152) holds. Using Lemma 29, it follows that

$$
\begin{aligned}
\operatorname{dim} W^{\omega} & =\operatorname{dim} V-\operatorname{dim} W+\operatorname{dim}\left(V^{\omega} \cap W\right) \\
& \leq \operatorname{dim} W^{i^{*} \omega}-\operatorname{dim} V^{\omega}+\operatorname{dim}\left(V^{\omega} \cap W\right) \\
& \leq \operatorname{dim} W^{i^{*} \omega} .
\end{aligned}
$$

Since $W^{\omega} \supseteq W^{i^{*} \omega}$, it follows that $W^{\omega}=W^{i^{*} \omega} \subseteq W$. Therefore, $W$ is coisotropic. This proves " $\Leftarrow$ " and completes the proof of Lemma 28 .

\section{References}

[Ab1] C. Abbas, A note on V. I. Arnold's chord conjecture. Internat. Math. Res. Notices 1999, no. 4, 217-222.

[Ab2] C. Abbas, Finite energy surfaces and the chord problem, Duke Math. J. 96 (1999), no. 2, 241-316.

[Ab3] C. Abbas, The chord problem and a new method of filling by pseudoholomorphic curves, Int. Math. Res. Not. 2004, no. 18, 913-927.

[Ar] V. I. Arnol'd, The first steps of symplectic topology, (Russian) Uspekhi Mat. Nauk 41 (1986), no. 6 (252), 3-18, 229.

[BC1] P. Biran, K. Cieliebak, Symplectic topology on subcritical manifolds, Comment. Math. Helv. 76 (2001), no. 4, 712-753.

[BC2] P. Biran, K. Cieliebak, Lagrangian embeddings into subcritical Stein manifolds, Israel J. Math. 127 (2002), 221-244.

[BEE] F. Bourgeois, T. Ekholm, and Y. Eliashberg, Effect of Legendrian surgery, with an appendix by S. Ganatra and M. Maydanskiy, Geom. Topol. 16 (2012), no. 1, 301-389.

[Ch1] Yu. Chekanov, Critical points of quasifunctions, and generating families of Legendrian manifolds, (Russian) Funktsional. Anal. i Prilozhen. 30 (1996), no. 2, 56-69, 96; translation in Funct. Anal. Appl. 30 (1996), no. 2, 118-128.

[Ch2] Yu. Chekanov, Lagrangian intersections, symplectic energy, and areas of holomorphic curves, Duke Math. J. 95 (1998), no. 1, 213-226.

[Ch3] Yu. Chekanov, Differential algebra of Legendrian links, Invent. Math. 150 (2002), no. 3, 441-483.

[Ci] K. Cieliebak, Handle attaching in symplectic homology and the chord conjecture, J. Eur. Math. Soc. 4 (2002), no. 2, 115-142.

$[\mathrm{CM}]$ K. Cieliebak and K. Mohnke, Punctured holomorphic curves and Lagrangian embeddings, arXiv:1411.1870v2.

[Ek] T. Ekholm, Rational SFT, linearized Legendrian contact homology, and Lagrangian Floer cohomology, Perspectives in analysis, geometry, and topology, 109-145, Progr. Math., 296, Birkhäuser/Springer, New York, 2012. 
[EESa] T. Ekholm, J. B. Etnyre, J. Sabloff, A duality exact sequence for Legendrian contact homology, Duke Math. J. 150 (2009), no. 1, 1-75.

[EESu] T. Ekholm, J. B. Etnyre, M. Sullivan, Legendrian contact homology in $P \times \mathbb{R}$, Trans. Amer. Math. Soc. 359 (2007), no. 7, 3301-3335.

[EGH] Y. Eliashberg, A. Givental, H. Hofer, Introduction to symplectic field theory, Geom. Funct. Anal. 2000, Special Volume, Part II, 560-673.

[GG] V. L. Ginzburg and B. Gürel, A $C^{2}$-smooth counterexample to the Hamiltonian Seifert Conjecture in $\mathbb{R}^{4}$, Ann. Math., Second Series, Vol. 158, no. 3 (2003), 953-976.

[Giv] A. B. Givental', The nonlinear Maslov index, Geometry of low-dimensional manifolds, 2 (Durham, 1989), 35-43, London Math. Soc. Lecture Note Ser. 151, Cambridge Univ. Press, Cambridge, 1990.

[HT1] M. Hutchings and C.H. Taubes, Proof of the Arnold chord conjecture in three dimensions, I, Math. Res. Lett. 18 (2011), no. 2, 295-313.

[HT2] M. Hutchings and C.H. Taubes, Proof of the Arnold chord conjecture in three dimensions, II, Geom. Topol. 17 (2013), no. 5, 2601-2688.

[Le] J. M. Lee, Introduction to smooth manifolds, second ed., Graduate Texts in Mathematics, 218. Springer, New York, 2013. xvi+708 pp.

[Me] W. J. Merry, Lagrangian Rabinowitz Floer homology and twisted cotangent bundles, Geom. Dedicata 171 (2014), 345-386.

[Mo] K. Mohnke, Holomorphic disks and the chord conjecture, Ann. of Math. (2) 154 (2001), no. 1, 219-222.

[Rit] A. Ritter, Topological quantum field theory structure on symplectic cohomology, J. Topol. 6 (2013), no. 2, 391-489.

[Sa1] S. Sandon, On iterated translated points for contactomorphisms of $R^{2 n+1}$ and $R^{2 n} \times S^{1}$, Internat. J. Math. 23 (2012), no. 2, 1250042, 14 pp.

[Sa2] S. Sandon, A Morse estimate for translated points of contactomorphisms of spheres and projective spaces, Geom. Dedicata 165 (2013), 95-110.

[Se] P. Seidel, Graded Lagrangian submanifolds, Bull. Soc. Math. France 128 (2000), no. 1, 103-149.

[SZ] J. Swoboda and F. Ziltener, Coisotropic Displacement and Small Subsets of a Symplectic Manifold, Math. Z., Vol. 271, Iss. 1 (2012), p. 415-445.

[Zi] F. Ziltener, Coisotropic Submanifolds, Leafwise Fixed Points, and Presymplectic Embeddings, J. Symplectic Geom. 8 (2010), no. 1, 1-24. 